\title{
Orientational dynamics in nematic liquid crystals
}

DOI:

10.1140/epjst/e2016-60118-1

\section{Document Version}

Accepted author manuscript

Link to publication record in Manchester Research Explorer

\section{Citation for published version (APA):}

Humpert, A., Masters, A., \& Allen, M. P. (2016). Orientational dynamics in nematic liquid crystals: A coarse-grained simulation study. The European Physical Journal. Special Topics, 225(8), 1723-1732.

https://doi.org/10.1140/epjst/e2016-60118-1

\section{Published in:}

The European Physical Journal. Special Topics

\section{Citing this paper}

Please note that where the full-text provided on Manchester Research Explorer is the Author Accepted Manuscript or Proof version this may differ from the final Published version. If citing, it is advised that you check and use the publisher's definitive version.

\section{General rights}

Copyright and moral rights for the publications made accessible in the Research Explorer are retained by the authors and/or other copyright owners and it is a condition of accessing publications that users recognise and abide by the legal requirements associated with these rights.

\section{Takedown policy}

If you believe that this document breaches copyright please refer to the University of Manchester's Takedown Procedures [http://man.ac.uk/04Y6Bo] or contact uml.scholarlycommunications@manchester.ac.uk providing relevant details, so we can investigate your claim.

\section{OPEN ACCESS}




\title{
Orientational dynamics in nematic liquid
}

\section{crystals}

\section{A coarse-grained simulation study}

Anja Humpert ${ }^{1, a}$, Andrew J. Masters ${ }^{2, b}$, and Michael P. Allen ${ }^{3,1, c, d}$

1 Department of Physics, University of Warwick, Coventry CV4 7AL, United Kingdom

2 School of Chemical Engineering and Analytical Science, The University of Manchester, Oxford Road, Manchester, M13 9PL, United Kingdom

${ }^{3}$ H. H. Wills Physics Laboratory, Royal Fort, Tyndall Avenue, Bristol BS8 1TL, United Kingdom

\begin{abstract}
We examine the behaviour of single-particle orientational time correlation functions in nematic liquid crystals. As well as the expected dynamics involving oscillation in a mean-field potential, and occasional jumps between orientations parallel and antiparallel to the director, we provide the first simulation evidence of long-time tails characteristic of coupling to director fluctuations.
\end{abstract}

\footnotetext{
a e-mail: a.humpert@warwick.ac.uk

b e-mail: andrew.masters@manchester.ac.uk

c e-mail: m.p.allen@bristol.ac.uk

d e-mail: m.p.allen@warwick.ac.uk
} 


\section{Introduction}

The nematic liquid crystal phase is characterized by long-ranged orientational order, but short-ranged positional order. There is a preferred direction in space, the director

$\mathbf{n}$, which breaks the rotational symmetry. Reorientational motion in nematics involves end-over-end tumbling, and libration, in the mean field provided by the neighbouring molecules. Accordingly, and bearing in mind that nematics are typically dense liquids, one might think that this can be described by a rotational diffusion model in an external, orientation-dependent, potential. However, things are not so simple. Firstly, rotational motion may be intrinsically collective in nature, involving clusters of molecules reorienting together. Secondly, although the director acts as a quasiconserved variable, it is subject to fluctuations over a range of wavelengths which typically affect the single-particle motion. In this paper, we study some aspects of this motion by molecular simulation using a coarse-grained model, concentrating on this last aspect.

As discussed in [1], the time evolution of single-particle orientations in liquid crystals may be coupled to fluctuations of the nematic director. For the appropriate component of the second-rank orientation tensor, a crude mode-coupling theory $[2,3$, and references therein] predicts a long-time tail of the form $t^{-1 / 2}$. This is supported by a more sophisticated theoretical analysis along the lines of [4] and by experiment $[5]$.

In the following sections we establish the notation used to describe rotational time correlation functions, and mention briefly the rotational diffusion model; then we present the simulation model and methods, followed by our results and some conclusions. 


\section{Theory}

In this paper we consider axially symmetric molecules, for which the unit orientation vectors are described in terms of Cartesian components or polar angles:

$$
\mathbf{u}=\left(u_{X}, u_{Y}, u_{Z}\right)=(\sin \theta \cos \phi, \sin \theta \sin \phi, \cos \theta)
$$

We adopt a coordinate system in which the nematic director $\mathbf{n}$ lies along the $Z$ axis.

The molecular orientations are used to calculate spherical harmonic functions $Y_{m}^{\ell}\left(\mathbf{u}_{t}\right)$, where $t$ is a time argument, and hence the time correlation functions of interest in the nematic phase. We use the Condon and Shortley phase convention, as described in $[6]$

$$
Y_{m}^{\ell}(\theta, \phi)=(-)^{m} \sqrt{\frac{2 \ell+1}{4 \pi}} \sqrt{\frac{(\ell-m) !}{(\ell+m) !}} P_{m}^{\ell}(\cos \theta) \exp (\mathrm{i} m \phi), \quad 0 \leq m \leq \ell,
$$

where $P_{m}^{\ell}(\cos \theta)$ are associated Legendre polynomials, and for $-\ell \leq m<0$ use

$$
Y_{-m}^{\ell}(\theta, \phi)=(-)^{m} Y_{m}^{\ell}(\theta, \phi)^{*}
$$

Hence

$$
Y_{-m}^{\ell}\left(\theta_{0}, \phi_{0}\right) Y_{m}^{\ell}\left(\theta_{t}, \phi_{t}\right) \propto P_{m}^{\ell}\left(\cos \theta_{0}\right) P_{m}^{\ell}\left(\cos \theta_{t}\right) \exp \left[\mathrm{i} m\left(\phi_{t}-\phi_{0}\right)\right]
$$

and averaging eliminates the imaginary part, giving the single-particle orientational correlation functions of interest to us

$$
\begin{aligned}
c_{m}^{\ell}(t) & \propto\left\langle Y_{-m}^{\ell}\left(\theta_{0}, \phi_{0}\right) Y_{m}^{\ell}\left(\theta_{t}, \phi_{t}\right)\right\rangle \\
& \propto\left\langle P_{m}^{\ell}\left(\cos \theta_{0}\right) P_{m}^{\ell}\left(\cos \theta_{t}\right) \cos m\left(\phi_{t}-\phi_{0}\right)\right\rangle \quad 0 \leq m \leq \ell .
\end{aligned}
$$

The proportionality constant is chosen such that $c_{m}^{\ell}(0)=1$. In an isotropic liquid, all the rank- $\ell$ functions $c_{m}^{\ell}(t)$ with $0 \leq m \leq \ell$ are identical. In the rotational diffusion approximation, in an isotropic liquid, we expect the functions for different $\ell$ to be related by

$$
c^{\ell}(t)=\exp \left[-\ell(\ell+1) D_{\mathrm{r}} t\right]
$$


where $D_{\mathrm{r}}$ is the rotational diffusion coefficient, given by

$$
D_{\mathrm{r}}^{-1}=\ell(\ell+1) \int_{0}^{\infty} \mathrm{d} t c^{\ell}(t) \quad \forall \ell .
$$

In this approximation, it is also possible to relate $D_{\mathrm{r}}$ to the normalized correlation function of angular velocities $\boldsymbol{\omega}$

$$
D_{\mathrm{r}}=\frac{k_{\mathrm{B}} T}{I} \int_{0}^{\infty} \mathrm{d} t c_{\omega}(t)=\frac{k_{\mathrm{B}} T}{I} \int_{0}^{\infty} \mathrm{d} t \frac{\left\langle\boldsymbol{\omega}_{0} \cdot \boldsymbol{\omega}_{t}\right\rangle}{\left\langle|\boldsymbol{\omega}|^{2}\right\rangle}
$$

where $T$ is the temperature, $k_{\mathrm{B}}$ Boltzmann's constant, and $I$ the moment of inertia. More sophisticated models of rotational motion exist $[7,8]$ but will not be discussed here.

In the nematic phase, however, the $c_{m}^{\ell}(t)$ functions with the same $\ell$ but different $m$ are in general different. The combination of $\pm m$ indices in eqn (4a) guarantees invariance with respect to rotation about the $Z$-axis. The $c_{0}^{\ell}(t)$ functions describe end-over-end rotation of the molecule in the orienting mean field provided by the surrounding molecules, which favour alignment along the director. The higher values of $m$ include contributions from reorientation about the director.

\section{Simulation details}

We use the interaction potential originally suggested by Gay and Berne [9], a coarsegrained single-site potential representing the interaction energies between two elongated (or disk-shaped) molecules. It can be regarded as a shifted, anisotropic LennardJones potential, i.e. it depends on the relative orientation of the particles as well as their separation. For identical uniaxial particles it can be written as $[10,11]$

$$
U_{\mathrm{GB}}\left(\mathbf{u}_{i}, \mathbf{u}_{j}, \mathbf{R}_{i j}\right)=4 \epsilon\left(\mathbf{u}_{i}, \mathbf{u}_{j}, \mathbf{r}_{i j}\right)\left[\varrho\left(\mathbf{u}_{i}, \mathbf{u}_{j}, \mathbf{R}_{i j}\right)^{-12}-\varrho\left(\mathbf{u}_{i}, \mathbf{u}_{j}, \mathbf{R}_{i j}\right)^{-6}\right],
$$

where

$$
\varrho\left(\mathbf{u}_{i}, \mathbf{u}_{j}, \mathbf{R}_{i j}\right)=\frac{R_{i j}-\sigma\left(\mathbf{u}_{i}, \mathbf{u}_{j}, \mathbf{r}_{i j}\right)+\sigma_{0}}{\sigma_{0}} .
$$


As before, $\mathbf{u}_{i}$ and $\mathbf{u}_{j}$ are unit vectors along the principal axes of the two particles $i$ and $j$, while $\mathbf{R}_{i j}=\mathbf{R}_{i}-\mathbf{R}_{j}$ is the vector connecting their centres of mass, $R_{i j}=\left|\mathbf{R}_{i j}\right|$, and $\mathbf{r}_{i j}=\mathbf{R}_{i j} / R_{i j} . \sigma_{0}$ is a parameter representing the width of the particle. $\sigma\left(\mathbf{u}_{i}, \mathbf{u}_{j}, \mathbf{r}_{i j}\right)$ is the orientation-dependent range parameter

$$
\sigma\left(\mathbf{u}_{i}, \mathbf{u}_{j}, \mathbf{r}_{i j}\right)=\sigma_{0}\left[1-\frac{\chi}{2}\left(\frac{\left(\mathbf{r}_{i j} \cdot \mathbf{u}_{i}+\mathbf{r}_{i j} \cdot \mathbf{u}_{j}\right)^{2}}{1+\chi \mathbf{u}_{i} \cdot \mathbf{u}_{j}}+\frac{\left(\mathbf{r}_{i j} \cdot \mathbf{u}_{i}-\mathbf{r}_{i j} \cdot \mathbf{u}_{j}\right)^{2}}{1-\chi \mathbf{u}_{i} \cdot \mathbf{u}_{j}}\right)\right]^{-1 / 2}
$$

Here $\chi$ is given by

$$
\chi=\frac{\kappa^{2}-1}{\kappa^{2}+1}
$$

where $\kappa$ is the length-to-width ratio of the particle. The strength anisotropy function $\epsilon\left(\mathbf{u}_{i}, \mathbf{u}_{j}, \mathbf{r}_{i j}\right)$ used in Equation (8) is given by

$$
\epsilon\left(\mathbf{u}_{i}, \mathbf{u}_{j}, \mathbf{r}_{i j}\right)=\epsilon_{0} \epsilon_{1}^{\nu}\left(\mathbf{u}_{i}, \mathbf{u}_{j}\right) \epsilon_{2}^{\mu}\left(\mathbf{u}_{i}, \mathbf{u}_{j}, \mathbf{r}_{i j}\right)
$$

$\epsilon_{0}$ is the well depth parameter determining the overall strength of the potential, while $\nu$ and $\mu$ are two adjustable exponents which allow considerable flexibility in defining a family of Gay-Berne potentials. $\epsilon_{1}$ and $\epsilon_{2}$ are given by

$$
\begin{gathered}
\left.\epsilon_{1}\left(\mathbf{u}_{i}, \mathbf{u}_{j}\right)=\left[1-\chi^{2}\left(\mathbf{u}_{i} \cdot \mathbf{u}_{j}\right)^{2}\right)\right]^{-1 / 2} \\
\epsilon_{2}\left(\mathbf{u}_{i}, \mathbf{u}_{j}, \mathbf{r}_{i j}\right)=1-\frac{\chi^{\prime}}{2}\left[\frac{\left(\mathbf{r}_{i j} \cdot \mathbf{u}_{i}+\mathbf{r}_{i j} \cdot \mathbf{u}_{j}\right)^{2}}{1+\chi^{\prime} \mathbf{u}_{i} \cdot \mathbf{u}_{j}}+\frac{\left(\mathbf{r}_{i j} \cdot \mathbf{u}_{i}-\mathbf{r}_{i j} \cdot \mathbf{u}_{j}\right)^{2}}{1-\chi^{\prime} \mathbf{u}_{i} \cdot \mathbf{u}_{j}}\right]
\end{gathered}
$$

Here

$$
\chi^{\prime}=\frac{\kappa^{\prime 1 / \mu}-1}{\kappa^{\prime 1 / \mu}+1}
$$

where $\kappa^{\prime}=\epsilon_{\mathrm{S}} / \epsilon_{\mathrm{E}}$ is the ratio of well depths for the side-to-side configuration, $\epsilon_{\mathrm{S}}$, and the end-to-end configuration, $\epsilon_{\mathrm{E}}$, of two molecules. Different versions of the potential are identified by the $\operatorname{GB}\left(\kappa, \kappa^{\prime}, \mu, \nu\right)$ notation of Bates and Luckhurst [12]. As in our previous work $[13,14]$ we have simulated $\mathrm{GB}(3,5,2,1)$, the original suggestion of Gay and Berne [9], for which the phase diagram has been well studied [15], and $\operatorname{GB}(3,5,1,3)$, proposed by Berardi et al. [10]. For each system we chose a single state point in the nematic phase (see Table 1). We used the simulation program GBMOLDD [16] which 
Table 1. Simulation details. For each system $\operatorname{GB}\left(\kappa, \kappa^{\prime}, \mu, \nu\right)$ with reduced moment of inertia $I=0.5$, at density $\rho$ and target temperature $T$, with the indicated system size $N$, we report: the average temperature $\langle T\rangle$; nematic order parameter $\langle S\rangle$; and the root-meansquare angular deviation $\theta_{\mathrm{rms}}$ (in radians) of the director from its average, over the whole production run of $10^{6}$ steps. Numbers in parentheses represent estimated errors in the last reported digit. Simulation units are defined in the text.

\begin{tabular}{lcccccc}
\hline system & \multicolumn{2}{c}{$\mathrm{GB}(3,5,2,1), \rho=0.32, T=0.90$} & \multicolumn{2}{c}{$\mathrm{GB}(3,5,1,3), \rho=0.30, T=3.40$} \\
$N$ & 8000 & 64000 & 512000 & 8000 & 64000 & 512000 \\
$L$ & 29.240 & 58.480 & 116.960 & 29.876 & 59.752 & 119.504 \\
\hline$\langle T\rangle$ & $0.9051(2)$ & $0.8992(1)$ & $0.9000(1)$ & $3.394(1)$ & $3.3968(3)$ & $3.4007(1)$ \\
$\langle S\rangle$ & $0.667(2)$ & $0.662(1)$ & $0.6590(4)$ & $0.616(1)$ & $0.607(1)$ & $0.6018(4)$ \\
$\theta_{\text {rms }}$ & 0.06 & 0.03 & 0.02 & 0.3 & 0.1 & 0.02 \\
\hline
\end{tabular}

employs a cut-and-shifted form of the potential

$$
U_{\mathrm{GB}}\left(\mathbf{u}_{i}, \mathbf{u}_{j}, \mathbf{R}_{i j}\right)-U_{\mathrm{GB}}\left(\mathbf{u}_{i}, \mathbf{u}_{j}, R_{\mathrm{c}} \mathbf{r}_{i j}\right)
$$

with spherical cutoff distance $R_{\mathrm{c}}=5 \sigma_{0}$. We adopt units in which $\sigma_{0}=1, \epsilon_{0}=1$, and the molecular mass $m_{0}=1$. This leads to a basic unit of time $\tau_{0}=\sigma_{0} \sqrt{m_{0} / \epsilon_{0}}$. All results reported here are referred to these units. The molecules were treated as linear rotors, with no rotation about the $\mathbf{u}$ axis, and a single moment of inertia $I$ corresponding to rotation about any axis perpendicular to $\mathbf{u}$. Most results were obtained using $I=0.5 m_{0} \sigma_{0}^{2}$, corresponding to uniform mass distribution within the ellipsoidal particle. All runs were carried out in the constant- $N V E$ microcanonical ensemble with a time step $\delta t=0.002$. Consequently, in all reported results, one time unit corresponds to 500 time steps. All production runs were of length $10^{6}$ steps, i.e. 2000 units of time. In order to study system size effects, we used 'small', $N=8000$, 'medium', $N=64000$, and 'large', $N=512000$ systems, chosen to make the linear 
dimensions of the periodic simulation box in the ratio 1:2:4. Simulation details are summarized in Table 1. A few comparison runs were conducted with an enhanced moment of inertia, $I=2.5 m_{0} \sigma_{0}^{2}$, for $N=64000$, using the same other parameters as above.

In a nematic phase we define an average order tensor $Q$ as

$$
Q_{\alpha \beta}=\frac{1}{N} \sum_{i=1}^{N}\left(\frac{3}{2} u_{i \alpha} u_{i \beta}-\frac{1}{2} \delta_{\alpha \beta}\right) .
$$

Here $\delta_{\alpha \beta}$ is the Kronecker delta, and we define Cartesian components $\alpha, \beta=x, y, z$ in the frame of the simulation box. The largest eigenvalue of $\mathrm{Q}$ is the order parameter $S$ and its corresponding normalized eigenvector represents the director $\mathbf{n}$. This is used to define the $Z$-direction in the Cartesian coordinate system described in section 2; the $X$ and $Y$ directions are defined perpendicular to $Z$ and to each other. One technical issue concerns director drift. Ideally, the $X Y Z$ system would remain fixed throughout the run; in practice this is not the case, and the director tends to reorient very slowly. It is possible to introduce a set of Lagrangian constraints to fix the director [17]. Here, we prefer to leave the equations of motion unmodified, and allow $Z$ to follow small variations in the director, while changes in $X$ and $Y$ are kept to the minimum necessary to ensure orthogonality with $Z$. This approach is justifiable for the medium and large systems, for which the root-mean-square variation of the director, even during the course of the very long runs undertaken here, amounts to no more than a few degrees (see table 1). However for the $N=8000$, specifically in the $\operatorname{GB}(3,5,1,3)$ system, the director drift is larger, and must be borne in mind when interpreting the results.

\section{Results}

In Fig. 1 we show the normalized angular velocity correlation function for the two systems of interest. This function decays rapidly due to the strong orientational caging 
in the nematic phase. The short time behaviour is given by

$$
\frac{\left\langle\boldsymbol{\omega}_{0} \cdot \boldsymbol{\omega}_{t}\right\rangle}{\left\langle|\boldsymbol{\omega}|^{2}\right\rangle}=1-\frac{1}{2} \frac{\left\langle|\dot{\boldsymbol{\omega}}|^{2}\right\rangle}{\left\langle|\boldsymbol{\omega}|^{2}\right\rangle} t^{2}+\mathcal{O}\left(t^{4}\right)=1-\frac{1}{4} \frac{\left\langle|\boldsymbol{\tau}|^{2}\right\rangle}{I k_{\mathrm{B}} T} t^{2}+\mathcal{O}\left(t^{4}\right)
$$

where $\left\langle|\boldsymbol{\tau}|^{2}\right\rangle$ is the mean-squared torque, and we use equipartition of energy to set $\left\langle|\boldsymbol{\omega}|^{2}\right\rangle=2 k_{\mathrm{B}} T / I$ for two degrees of rotational freedom. Accordingly, plots of the correlation function against $t / \sqrt{I}$ should be superimposable at short times, and this is illustrated in Fig. 1. However, even on this plot, functions corresponding to the different moments of inertia diverge quite quickly from one another; the negative minimum and long-time approach to zero for $I=2.5$ occur on a timescale roughly $3.5 \times$ that of the $I=0.5$ system, in both cases.

In Fig. 2 we show plots of the orientational correlation functions $c_{0}^{\ell}(t)$ corresponding to end-over-end rotation. At short times, all the functions decay from their initial values, with the higher-ranked functions varying most quickly. The curves, however, are not particularly well fitted by exponential functions of the time, and do not superimpose well if the time is scaled according to $t \rightarrow \ell(\ell+1) t$, and so a simple rotational diffusion picture is not appropriate. Instead, the dominant motion is likely to be libration inside the mean-field orientational potential provided by the surrounding nematic. The even- $\ell$ functions quite rapidly equilibrate to the non-zero long-time values dictated by the nematic ordering:

$$
\lim _{t \rightarrow \infty} c_{0}^{\ell}(t)=\frac{\left\langle P^{\ell}(\cos \theta)\right\rangle^{2}}{\left\langle P^{\ell}(\cos \theta)^{2}\right\rangle}= \begin{cases}\frac{S_{2}^{2}}{\frac{1}{5}+\frac{2}{7} S_{2}+\frac{18}{35} S_{4}} & \ell=2 \\ \frac{S_{4}^{2}}{\frac{1}{9}+\frac{100}{693} S_{2}+\frac{162}{1001} S_{4}+\frac{20}{99} S_{6}+\frac{490}{1287} S_{8}} & \ell=4\end{cases}
$$

where $S_{\ell}=\left\langle P^{\ell}(\cos \theta)\right\rangle$. The odd- $\ell$ functions, on the other hand, decay slowly, and exponentially, towards zero. This decay is essentially independent of $\ell$, and is characterized by a time $t_{\text {jump }}$ between end-over-end jumps which invert the molecular orientation $\mathbf{u} \rightarrow-\mathbf{u}$. This time is strongly influenced by the free energy barrier occurring when the molecular orientation is perpendicular to the director. For the $\operatorname{GB}(3,5,2,1)$ 
$\mathrm{GB}(3,5,2,1)$

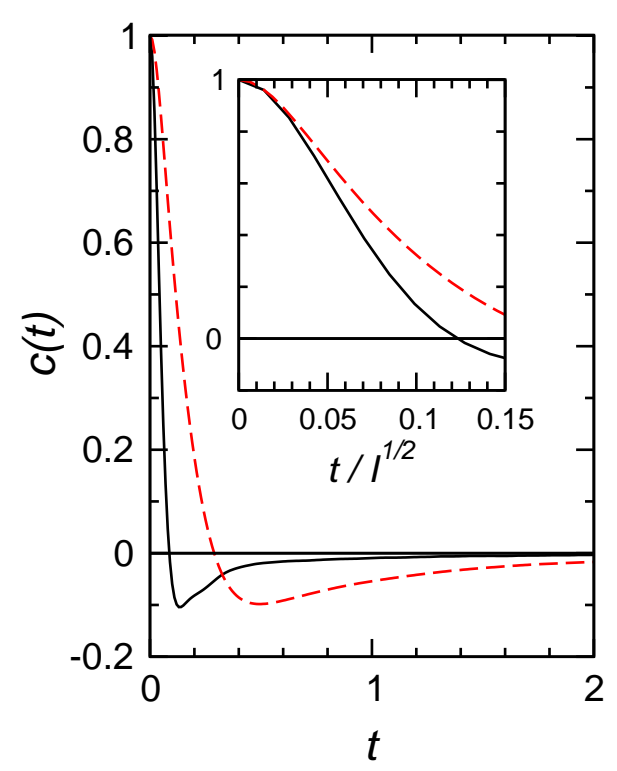

$\mathrm{GB}(3,5,1,3)$

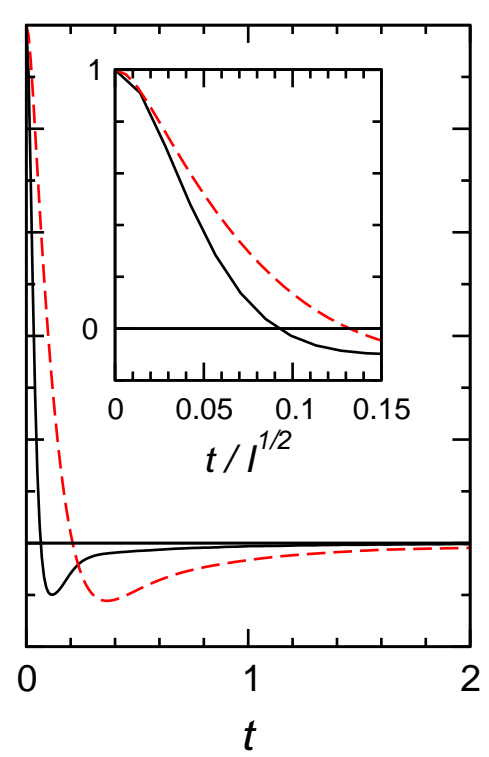

Fig. 1. Angular velocity correlation functions for the systems under study, for $N=64000$.

(These results are almost independent of system size). Solid (black) lines correspond to $I=0.5$, dashed (red) lines to $I=2.5$. The insets show the same functions plotted against $t / \sqrt{I}$ to illustrate the initial quadratic decay, which is determined by the mean-squared torque. Estimated errors are comparable with the line width.

system a least-squares fit gives $t_{\text {jump }}=216 \pm 1(I=0.5)$ and $t_{\text {jump }}=271 \pm 1(I=2.5)$; for the $\operatorname{GB}(3,5,1,3)$ system, the jumps are much more frequent: $t_{\text {jump }}=30 \pm 0.5$ $(I=0.5)$ and $t_{\text {jump }}=41.5 \pm 0.5(I=2.5)$. The $\mathrm{GB}(3,5,1,3)$ system has a somewhat smaller nematic order parameter, which is consistent with these observations. This type of motion may be associated with dielectric relaxation in nematics [18] and it is possible to relate the activation barrier for jumping to the orientational distribution and hence to the order parameter. However, as pointed out by de Gennes [19], the reorientation may not be strictly a single-molecule process, and so we postpone further investigation of this for later. 


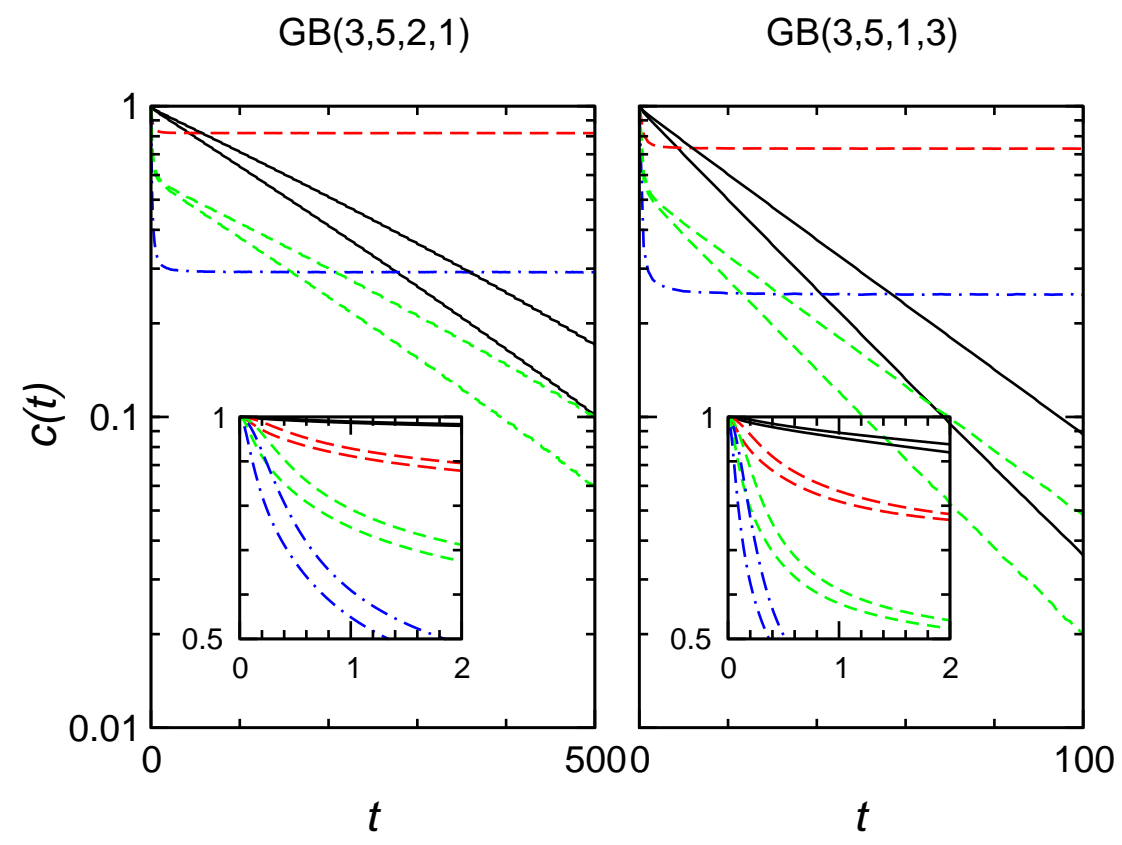

Fig. 2. Orientational correlation functions $c_{0}^{\ell}(t)$ for end-over-end rotation, plotted on a loglinear scale, for $N=64000$, (These results are almost independent of system size). Solid (black) lines: $\ell=1$; long-dashed (red) lines: $\ell=2$; short-dashed (green) lines: $\ell=3$; dotdashed (blue) lines: $\ell=4$. We show results for both $I=0.5$ (faster-decaying curves) and $I=2.5$ (slower-decaying curves). The insets show the same functions plotted on linear scales at short times. The $I=2.5$ curves for even $\ell$ are omitted in the main graphs for clarity (they approach the same constant values as $I=0.5$ on this time scale). Estimated errors are comparable with the line width.

Now, to set the scene for what follows, we compare in Fig. 3 the different correlation functions for $\ell=2$. The essential feature is that both $c_{1}^{2}(t)$ and $c_{2}^{2}(t)$ decay to zero as $t \rightarrow \infty$ (in contrast to $\left.c_{0}^{2}(t)\right)$ but in qualitatively different ways. The decay of $c_{2}^{2}(t)$ is rapid, and approximately exponential. In contrast, $c_{1}^{2}(t)$ exhibits a slower decay than exponential, and we turn to this in detail next. Although we do not show it, this behaviour is shared by $c_{1}^{4}(t)$, and we expect all even- $\ell$ functions with $m=1$ to couple in a similar way to director fluctuations at all wave-numbers, producing similar 
$\mathrm{GB}(3,5,2,1)$

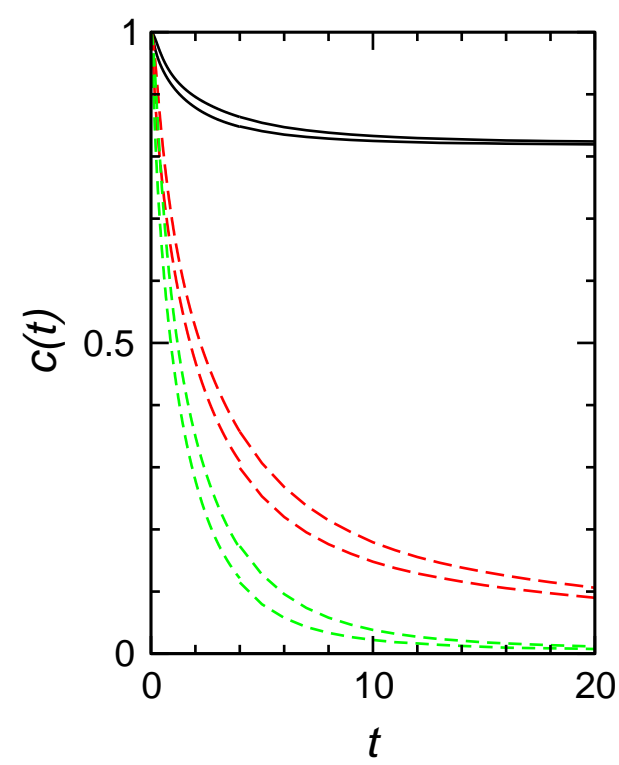

$\mathrm{GB}(3,5,1,3)$

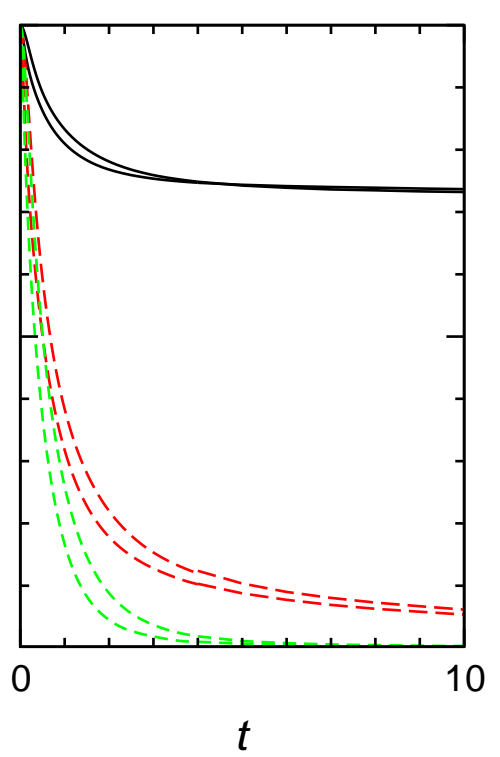

Fig. 3. Orientational correlation functions $c_{m}^{\ell}(t)$ plotted on a log-linear scale, for $N=64000$. Solid (black) lines: $c_{0}^{2}(t)$; long-dashed (red) lines: $c_{1}^{2}(t)$; short-dashed (green) lines: $c_{2}^{2}(t)$. Estimated errors are comparable with the line width. Faster-decaying curves correspond to $I=0.5$, slower-decaying ones to $I=2.5$.

algebraic long-time tails. Mode-coupling theory [2-4] predicts a long-time tail of the form $t^{-1 / 2}$. To see this in more detail, these functions are plotted in Fig. 4 for the two systems of interest on $\log -\log$ scales. In this case, a study of system size dependence is illuminating. The plots provide strong evidence for a limiting algebraic long-time tail, but this is moderated by an exponential decay which becomes more important for smaller system sizes. This would be expected if the single particle motion couples to director fluctuations of wave-number $k$, whose timescales vary as $\exp \left(-\lambda k^{2} t\right)$, where $\lambda$ is a transport coefficient related to orientational elastic constants and Leslie coefficients. Such behaviour is exactly what is predicted by standard nematodynamics [20-24], and we have explicitly verified it by molecular simulation $[13,14]$. The 
$\mathrm{GB}(3,5,2,1)$

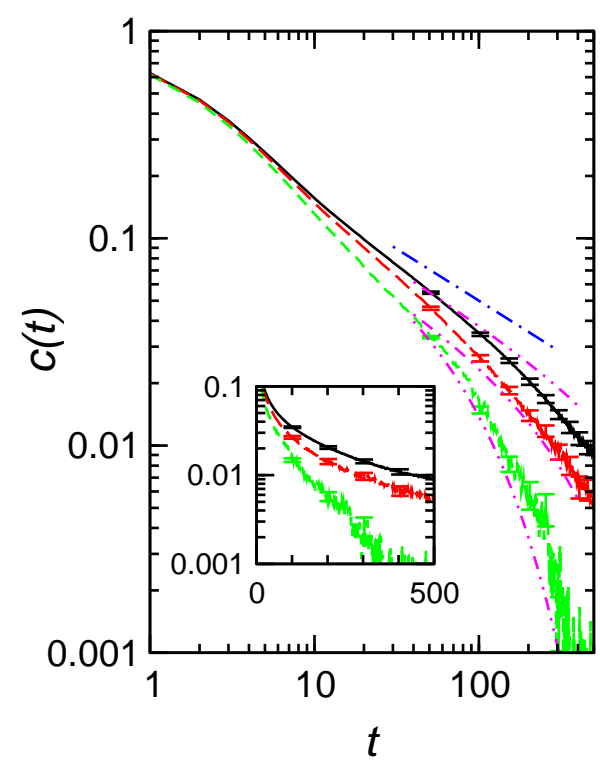

$\mathrm{GB}(3,5,1,3)$

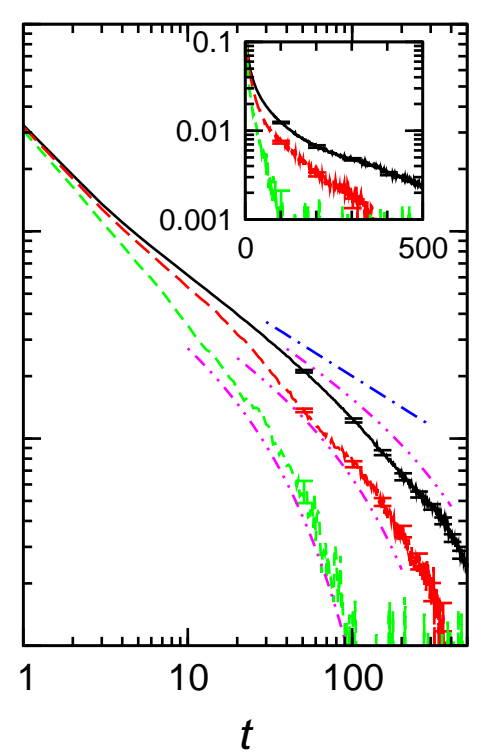

Fig. 4. Orientational correlation functions $c_{1}^{2}(t)$ plotted on a log-log scale, for the two systems of interest with $I=0.5$. We show results for different system sizes: solid (black) lines: $N=512000$; long-dashed (red) lines: $N=64000$; short-dashed (green) lines: $N=8000$. Estimated statistical uncertainties are indicated by error bars on selected points. In the insets we plot the same data on log-linear scales to investigate the exponential decay at long times. Also shown as guides to the eye are: dot-dashed (blue) lines: the algebraic decay $\propto t^{-1 / 2}$; double-dot-dashed (magenta) lines: the expected finite-size cutoff form, eqn (18), for each system size, with a single parameter $\lambda \approx 0.2$ for $\operatorname{GB}(3,5,2,1)$ and $\lambda \approx 0.6$ for $\operatorname{GB}(3,5,1,3)$. The vertical position of these guide lines is arbitrary: we have slightly offset them from the data for clarity.

mode-coupling theory relies on an integration over all such modes. In a finite-size system, there is a lower limit $k_{\min }=2 \pi / L$ imposed by the box length. Consequently, we anticipate that the $t^{-1 / 2}$ tail will be cut off by a decaying exponential giving an overall form

$$
c_{1}^{2}(t) \propto t^{-1 / 2} \exp \left(-\lambda k_{\min }^{2} t\right)
$$


Although the data at long times is quite noisy, especially for the $N=8000$ systems, $\log$-linear plots (see Fig. 4 insets) suggest that there is indeed an exponential cutoff. Because of the noise, and because of the director drift for $N=8000$, we do not attempt to extract the parameter $\lambda$ directly from our results. Instead, we have drawn guide lines in Fig. 4 corresponding to eqn (18) with parameters $\lambda \approx 0.2$ for $\operatorname{GB}(3,5,2,1)$ and $\lambda \approx 0.6$ for $\operatorname{GB}(3,5,1,3)$. Both these values are extremely close to the values reported for the hydrodynamic decay of splay and twist deformations at these state points [13]: $\left(\lambda_{\text {splay }}, \lambda_{\text {twist }}\right)=(0.15,0.17)$ for $\mathrm{GB}(3,5,2,1)$ and $(0.64,0.66)$ for $\mathrm{GB}(3,5,1,3)$. These modes are the slowest-varying ones; the bend mode decays by a factor 3-9 faster, and is also oscillatory. It is not possible to make a closer quantitative comparison, since the simple mode-coupling theory assumes isotropy in the elastic constants and transport coefficients. We note that, if the $t^{-1 / 2}$ tail persists in the limit of infinite system size, the integral of eqn (6) which usually defines an inverse rotational diffusion coefficient would diverge, and so $D_{\mathrm{r}}=0$ for $c_{1}^{2}(t)$.

\section{Conclusions}

In this work we have examined several aspects of single-particle reorientation in nematic liquid crystals. The angular velocity correlation function typically decays quickly, due to the strong caging effects of the neighbouring molecules. The orientational correlation functions characterizing end-over-end rotation show the expected behaviour: even-rank functions approach, quite quickly, a non-zero limiting value reflecting the non-vanishing orientational order parameters in this phase. On the other hand, odd-ranked functions decay very slowly to zero, due to infrequent rotations between the stable configurations parallel and antiparallel to the director. Finally, we have shown for the first time, by simulation, evidence in support of a long-time $t^{-1 / 2}$ tail in the function $c_{1}^{2}(t)$, due to coupling with hydrodynamic director fluctua- 
tion modes. The situation is complicated by the long-time exponential cutoff which is expected to result from finite size effects; however, our studies of different system sizes are consistent with this effect, and with the magnitudes of the relevant transport coefficients, as determined in a previous study [13]. Assuming that the long-time tail persists in the limit of infinite system size, it would be impossible to define a rotational diffusion coefficient from the $c_{1}^{2}(t)$ orientational correlations. It would be interesting to take this study further, and obtain a more precise correspondence between the collective and single-particle dynamics. Before doing so, however, it will be necessary to re-examine the underpinning mode-coupling theory [2-4], so as to relax the usual assumptions of a single elastic constant, and orientationally averaged transport coefficients.

Computer facilities were provided by Warwick University Centre for Scientific Computing. Support from the Engineering and Physical Sciences Research Council is gratefully acknowledged. We are grateful to Jaroslav Ilnytskyi for discussions regarding the GBMOLDD program.

This paper is dedicated to Kurt Kremer on the occasion of his 60th birthday. May there be many more years of inspiration in soft matter simulation ahead!

\section{References}

1. A.J. Masters, Molec. Phys. 95, 251 (1998)

2. P. Ukleja, J. Pirs, J.W. Doane, Phys. Rev. A 14, 414 (1976)

3. J.H. Freed, J. Chem. Phys. 66, 4183 (1977)

4. A. Masters, T. Keyes, J. Stat. Phys. 36, 401 (1984)

5. P.R. Luyten, J. Bulthuis, W.M.M.J. Bovée, L. Plomp, J. Chem. Phys. 78, 1712 (1983)

6. C.G. Gray, K.E. Gubbins, Theory of molecular fluids. 1. Fundamentals (Clarendon Press, Oxford, 1984), ISBN 0-19-855602-0

7. W.A. Steele, Adv. Chem. Phys. 34, 1 (1976) 
8. R.M. Lynden-Bell, in Molecular liquids: dynamics and Interactions, edited by A.J. Barnes, W.J. Orville-Thomas, J. Yarwood (D. Reidel, 1984), Vol. 135 of NATO ASI Series $C$, pp. 501-518

9. J.G. Gay, B.J. Berne, J. Chem. Phys. 74, 3316 (1981)

10. R. Berardi, A.P.J. Emerson, C. Zannoni, J. Chem. Soc. Faraday Trans. 89, 4069 (1993)

11. D.J. Cleaver, C.M. Care, M.P. Allen, M.P. Neal, Phys. Rev. E 54, 559 (1996)

12. M.A. Bates, G.R. Luckhurst, J. Chem. Phys. 110, 7087 (1999)

13. A. Humpert, M.P. Allen, Phys. Rev. Lett. 114, 028301 (2015)

14. A. Humpert, M.P. Allen, Molec. Phys. 113, 2680 (2015)

15. E. de Miguel, Molec. Phys. 100, 2449 (2002)

16. J.M. Ilnytskyi, M.R. Wilson, Comput. Phys. Commun. 134, 23 (2001)

17. M.P. Allen, M.A. Warren, M.R. Wilson, A. Sauron, W. Smith, J. Chem. Phys. 105, 2850 (1996)

18. G. Meier, A. Saupe, Mol. Cryst. 1, 515 (1966)

19. P.G. de Gennes, J. Prost, The Physics of Liquid Crystals, second, paperback edn. (Clarendon Press, Oxford, 1995)

20. J.L. Ericksen, Arch. Rational Mech. Anal. 4, 231 (1960)

21. F. Leslie, Arch. Rational Mech. Anal. 28, 265 (1968), ISSN 0003-9527

22. O. Parodi, J. de Physique 31, 581 (1970), ISSN 0302-0738

23. D. Forster, T.C. Lubensky, P.C. Martin, J. Swift, P.S. Pershan, Phys. Rev. Lett. 26, $1016(1971)$

24. P.C. Martin, O. Parodi, P.S. Pershan, Phys. Rev. A 6, 2401 (1972) 